\section{WUSCHEL protein movement mediates stem cell homeostasis in the Arabidopsis shoot apex}

\author{
Ram Kishor Yadav, ${ }^{1,3,4}$ Mariano Perales, ${ }^{1,3}$ \\ Jérémy Gruel, ${ }^{2}$ Thomas Girke, ${ }^{1}$ Henrik Jönsson, ${ }^{2}$ \\ and G. Venugopala Reddy ${ }^{1,5}$ \\ ${ }^{1}$ Department of Botany and Plant Sciences, Center for Plant Cell \\ Biology (CEPCEB), Institute of Integrative Genome Biology \\ (IIGB), University of California at Riverside, Riverside, \\ California 92521, USA; ${ }^{2}$ Computational Biology and Biological \\ Physics Group, Department of Astronomy and Theoretical \\ Physics, Lund University, SE-223 62 Lund, Sweden
}

WUSCHEL (WUS) is a homeodomain transcription factor produced in cells of the niche/organizing center (OC) of shoot apical meristems. WUS specifies stem cell fate and also restricts its own levels by activating a negative regulator, CLAVATA3 (CLV3), in adjacent cells of the central zone (CZ). Here we show that the WUS protein, after being synthesized in cells of the $\mathrm{OC}$, migrates into the $\mathrm{CZ}$, where it activates $C L V 3$ transcription by binding to its promoter elements. Using a computational model, we show that maintenance of the WUS gradient is essential to regulate stem cell number. Migration of a stem cell-inducing transcription factor into adjacent cells to activate a negative regulator, thereby restricting its own accumulation, is a theme that is unique to plant stem cell niches.

Supplemental material is available for this article.

Received July 5, 2011; revised version accepted September 2, 2011.

Cell-cell communication between distinct cell types within stem cell niches is critical for stem cell maintenance in both plants and animals, although they differ in their niche architecture and cell behaviors (Spradling et al. 2008; Rieu and Laux 2009). In animal systems-for example, the Drosophila germline-stem cells make direct contact with the niche cells through adherens junctions and receive local signals that prevent their differentiation (Spradling et al. 2008). Oriented and asymmetric division of stem cells places one of the progeny at a distance by a cell diameter, which no longer can receive signals from the niche and differentiates; thus, precise local cell behaviors regulate stem cell number. Whereas in the shoot apical meristem (SAM) stem cell niche not all stem cells make contact with the niche, they also do not exhibit oriented and asymmetric cell divisions to regulate stem cell numbers. For example, the Arabidopsis SAM stem cell niche

[Keywords: CLAVATA3; shoot apical meristem; CLAVATA1; central zone; niche]

${ }^{3}$ These authors contributed equally to this work.

${ }^{4}$ Present address: Faculty of Life Sciences and Biotechnology, South Asian University, JNU Campus, New Delhi 110067, India.

${ }^{5}$ Corresponding author.

E-mail venug@ucr.edu.

Article is online at http://www.genesdev.org/cgi/doi/10.1101/gad.17258511. is a collection of $\sim 500$ cells located at the growing tip of each shoot (Reddy 2008). The CZ of the SAM harbors stem cells. The stem cell progeny that are displaced into the adjacent peripheral zone (PZ) proliferate before differentiating (Fig. 1A; Reddy 2008). Visually, the SAM stem cell niche is a multilayered structure consisting of three clonally distinct layers of cells, and stem cells are found in each of these layers. The cells in the L1 and the L2 layers divide parallel to the surface to remain as monolayers. The cells in the L3 layer divide in random orientations to form a multilayered structure referred to as the rib meristem (RM) or the organizing center (OC), which provides stem cell-promoting cues (Rieu and Laux 2009).

Previous studies have shown that WUSCHEL (WUS), a homeodomain-containing transcription factor, is both necessary and sufficient for stem cell specification (Laux et al. 1996). WUS RNA is found in a few cells of the RM/OC located just beneath the CZ (Fig. 1A; Mayer et al. 1998). Restriction of WUS transcription to cells of the OC is critical for maintaining a constant number of stem cells, and this is mediated by the CLAVATA (CLV) signaling pathway (Mayer et al. 1998; Fletcher et al. 1999; Brand et al. 2000). CLAVATA3 (CLV3), expressed in the CZ, encodes a small peptide that is secreted into the extracellular space and binds to CLAVATA1 (CLV1), a leucinerich repeat receptor kinase predominantly expressed in cells of the RM (Clark et al. 1997; Fletcher et al. 1999; Kondo et al. 2006; Ogawa et al. 2008). Activation of CLV1 and related receptor kinases (Müller et al. 2008; Kinoshita et al. 2010) has been shown to mediate repression of WUS transcription through a signaling cascade that is not well understood. WUS, which is expressed in cells of the RM/ OC, not only specifies stem cell fate in overlying cells of the $\mathrm{CZ}$, but also activates its own negative regulator, CLV3, in a non-cell-autonomous manner. Thus, the WUSCLV feedback system forms a self-correcting mechanism for maintaining a constant number of stem cells and the SAM size.

A live-imaging study has shown that the transient downregulation of $C L V 3$ results in sequential expansion of $C L V 3$ promoter activity and of the CZ cells, suggesting that WUS could activate a diffusible stem cell-promoting signal (Reddy and Meyerowitz 2005). This apart, an earlier study has revealed that a transient increase in WUS levels not only results in expansion of the CZ, but also leads to an increase in cell division rates in the stem cell progenitors located in the PZ (Muller et al. 2006; Yadav et al. 2010). Thus, WUS, expressed in as few as 25-30 cells of the RM/ $\mathrm{OC}$, performs both the short- and the long-range functions that span across the $\mathrm{CZ}$ and the PZ. Gaining insights into the non-cell-autonomous functions of WUS requires identification of stem cell-promoting signals that emanate from the RM/OC, and also an understanding of the mechanism of communication between the RM/OC and rest of the SAM.

\section{Results and Discussion}

WUS protein migrates from OC/niche into adjacent cells

Intercellular movement of transcription factors forms an important mode of cell-cell communication in several 
Yadav et al.

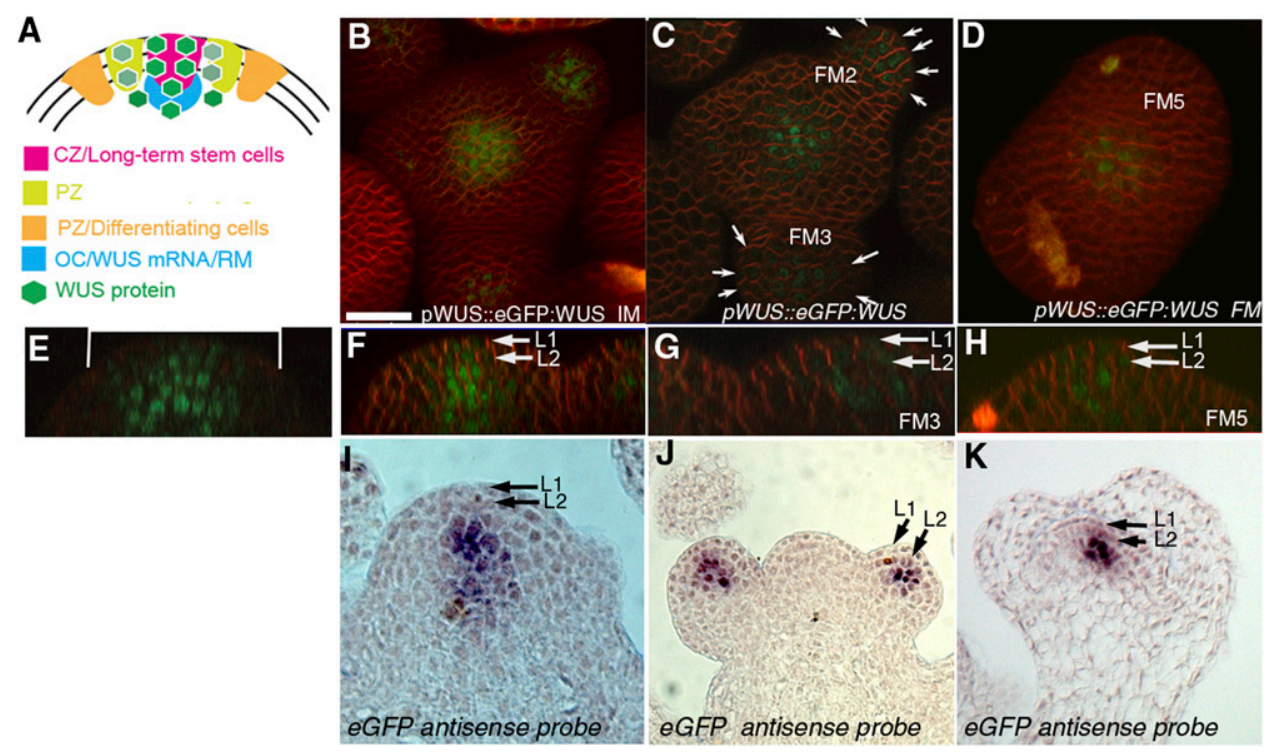

Figure 1. WUS protein movement. (A) A sketch of the Arabidopsis shoot apex showing various cell types compared with domains of WUS RNA and protein distribution. 3D reconstructed top view of a wus-1 mutant SAM rescued by $p W U S: \because e G F P$-WUS (B) and FMs of stage 2 (C), stage 3 $(C)$, and stage $5(D)$ showing eGFP-WUS protein (in green); cell outlines were stained with FM4-64 (red). (E, F) Side views of SAMs showing eGFP:WUS protein extending into the PZ and into the L1 layer. $(G, H)$ Side views of early and late stage FMs showing WUS protein localization. $I$, $I$, and $K$ are side views of SAMs, early flowers, and late flowers, respectively, showing eGFP mRNA distribution in $p W U S:: e G F P$ :WUSexpressing plants. Bar, $20 \mu \mathrm{M}$.

plant developmental processes (Gallagher and Benfey 2005). To test the possibility of cell-to-cell movement, we examined WUS protein localization. We generated a chimeric WUS protein by fusing eGFP (enhanced green fluorescent protein) protein-coding sequences with the $\mathrm{N}$ terminus of the WUS ORF. This fusion construct was expressed from the native WUS promoter, $p W U S:: e G F P: W U S$. After transformation into the wus-1 mutant background, this construct was able to rescue the mutant phenotype, confirming that it is functional (see the Materials and Methods; Supplemental Fig. S1A) and the eGFP:WUS fusion protein junction was not cleaved (Supplemental Fig. S1G,H). We observed a brighter fluorescence signal in cells of the $\mathrm{RM} / \mathrm{OC}$ where WUS is expressed and a relatively weaker signal in adjacent cells that extended up to the L1 layer of SAMs and into the PZ (Fig. 1B,E,F). The eGFP mRNA distribution in tissue sections of $p W U S$ ::eGFP:WUS-expressing plants revealed that $e G F P$ transcripts are localized to the L3 and deeper layers of the SAMs (Fig. 1I), very similar to the native WUS expression pattern described earlier (Mayer et al. 19981, excluding the possibility of WUS promoter misexpression from these constructs. A comparison of eGFP:WUS fluorescence with the GFP RNA in situ pattern in cells of the top-most L3 layer revealed that the WUS protein moves laterally by at least two cell layers (Supplemental Table S1). De novo stem cell specification occurs in early stages of floral meristem (FM) development, and the CLV-WUS feedback loop has been shown to mediate stem cell homeostasis in FMs. It has been shown that WUS transcripts are restricted to the L2 and deeper cell layers of FMs (Mayer et al. 1998). We observed a WUS protein gradient in FMs that extended up to the L1 layer of both early and late stage FMs (Fig. 1G,H). In early FMs, protein was detected in a much broader radial domain (Fig. 1C) than the eGFP mRNA distribution (Fig. 1J), whereas in late stage FMs, the radial spread of the WUS protein was limited to fewer cells (Fig. 1D). Taken together, the above observations reveal that WUS protein synthesized in cells of the RM/OC of SAMs and FMs migrates to adjacent cells.

\section{WUS protein migration is required for shoot meristem function}

Next, we tested whether WUS protein movement is required for its function. It has been shown that efficient targeting of plant transcription factors to the nucleus restricts their movement between cells (Crawford and Zambryski 2000; Gallagher et al. 2004). We generated a chimeric WUS protein in which eGFP containing a nuclear localization tag was fused to the $\mathrm{N}$ terminus of WUS and expressed from a native WUS promoter, $p W U S:: n l s-$ GFP:WUS. Nls-GFP:WUS fusion protein was found in the L2 layers of SAMs and in fewer cells in the radial domain (Fig. 2H; Supplemental Fig. 1I) in comparison with the eGFP:WUS protein distribution (Fig. 2B), showing that addition of a nuclear localization signal (nls) inhibits WUS protein movement. In early stage FMs, the fusion protein was detected in the L1 layer (Fig. 2K) and in a broader domain (Fig. 2J), similar to the eGFP:WUS distribution (Fig. 2D). However, in late stage FMs, nls-GFP:WUS was restricted to deeper cell layers and to fewer cell layers in the radial domain, revealing inhibition of protein movement (Fig. 2L). Plant transcription factors have been shown to move between cells through plasmodesmata, plasma membrane-lined channels (Crawford and Zambryski 2000). Plasmodesmata have been shown to have a size exclusion limit (SEL), allowing passage only to those molecules whose size falls below their SEL (Crawford and Zambryski 2000). To test this, we generated a chimeric WUS protein of higher molecular weight by fusing two tandem repeats of eGFP with the $\mathrm{N}$ terminus of WUS, $p W U S:: 2 X e G F P: W U S$. 


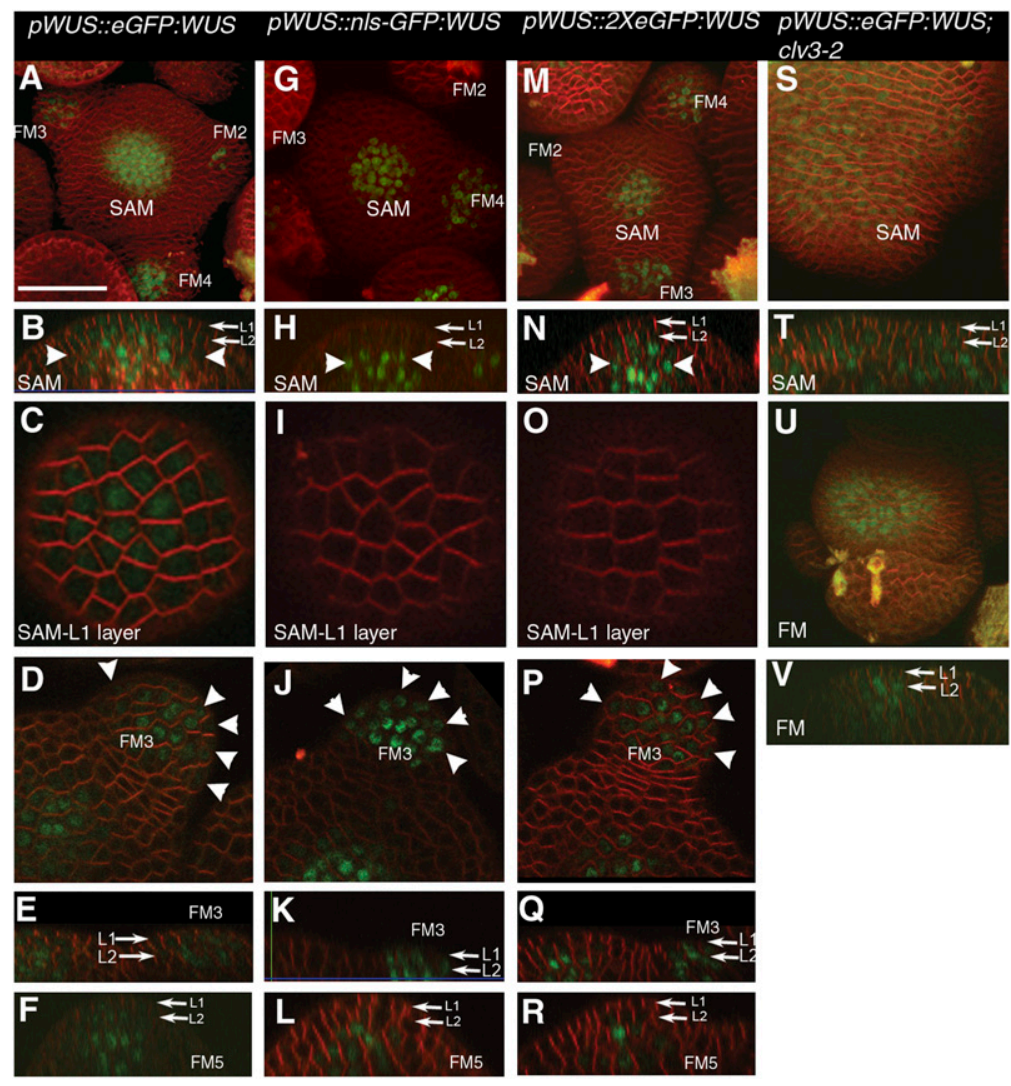

Figure 2. WUS protein movement is required for its function. WUS protein localizations of $p W U S:: e G F P: W U S(A-F)$, pWUS::nls-GFP:WUS $(G-L)$, and pWUS::2XGFP:WUS (M-R). (S-V) pWUS::eGFP:WUS in clv3-2 mutants. (Green) WUS protein; (red) cell membranes. $A, G, M$, and $S$ are $3 \mathrm{D}$ reconstructed top views of SAMs. $C, I$, and $O$ are confocal cross-sections showing the L1 layer of SAMs. $B, E, F$, $H, K, L, N, Q, R, T$, and $V$ are reconstructed side views of SAMs and FMs. (FM3) Stage 3 flower; (FM4) stage 4 flower); (FM5) stage 5 flower. Arrows point to the L1 layer (L1) and the L2 layer (L2). Opposing arrows in $B, H$, and $N$ indicate the width of WUS protein domains. Arrowheads in $D, I$, and $P$ indicate the radial domain of WUS expression in FM3. Bars: $C, I, O, 10 \mu \mathrm{M}$; all other panels, $35 \mu \mathrm{M}$.

This fusion protein failed to move beyond the L2 layer of SAMs (Fig. 2N) and was detected in fewer cells in the radial domain (Fig. 2N). In early stage FMs, the protein was observed in the L1 layer (Fig. 2Q) and in a broader domain (Fig. 2P), similar to the eGFP:WUS and nls-GFP:WUS. However, in late stage FMs, the 2XeGFP:WUS expression was restricted to deeper layers, showing inhibition of WUS protein movement (Fig. 2R). Both pWUS::nls-GFP:WUS and $p W U S:: 2 X e G F P: W U S$ failed to rescue the SAM phenotype of wus-1 mutants (Supplemental Fig. S1B,C). However, in a few cases, they developed axillary shoot meristems, which produced five to six flowers that contained a complete set of organs (see the Materials and Methods; Supplemental Fig. S1D,E). wus-1 mutants never produce complete flowers, and the rescue of flower phenotype reveals that the fusion proteins retain their biological activity. These results are consistent with the inhibition of movement of nls-GFP and 2XeGFP in SAMs and the lack of inhibition observed in the early stages of FMs. Taken together, these results show that inhibition of WUS protein movement does not interfere with its activity but interferes with SAM function, demonstrating that WUS protein movement is critical for SAM function.
It has been shown that CLV-mediated signaling negatively regulates $W U S$ transcription to restrict its expression to a few cells of the OC (Fletcher et al. 1999; Schoof et al. 2000). In clv3 mutants, a massive expansion of the $\mathrm{CZ}$ and $C L V 3$ promoter expression have been observed, along with the expansion of the WUS domain in underlying cell layers of SAMs and FMs (Fletcher et al. 1999; Schoof et al. 2000; Reddy and Meyerowitz 2005). To examine WUS protein distribution in these enlarged meristems, we introduced $p W U S:: e G F P: W U S$ into $c l v 3-2$ mutants. WUS protein was detected in the L1 layer cells and also expanded radially in clv3-2 mutant SAMs (Fig. 2S,T; Supplemental Fig. S1F) and FMs (Fig. 2U,V), suggesting that expansion of the CZ and the $C L V 3$ promoter activity may be a consequence of the presence of the WUS protein in these cells.

\section{WUS binds to CLV3 regulatory regions to activate its transcription}

In light of WUS protein movement into superficial cell layers of the CZ, we next examined the possibility of WUS activating CLV3 transcription directly. To test whether WUS is capable of activating CLV3 expression, we used a dexamethasone (Dex)-inducible form of WUS, 35S::WUS-GR. This construct was generated by fusing the WUS protein-coding region with the ligand-binding domain of the rat glucocorticoid receptor (GR) and was expressed from a ubiquitous promoter. Quantitative RT-PCR (qRT-PCR) analysis on RNA samples extracted from carefully dissected wild-type SAMs expressing $35 S:: W U S: G R$ showed that $C L V 3$ was activated within $4 \mathrm{~h}$ of Dex application in both the absence and presence of cycloheximide (Cyc), a protein synthesis inhibitor. This result suggests that $C L V 3$ may be a direct transcriptional target of WUS (Fig. 3A).

To test whether WUS directly binds to the promoter regions of $C L V 3$, we performed chromatin immunoprecipitation (ChIP) coupled to qPCR analysis (ChIPqPCR) by using anti-WUS (peptide) antibodies (Supplemental Fig. S2A,B). After screening different promoter regions of $C L V 3$, we obtained immunoprecipitated DNA that was enriched in sequences located upstream of and downstream from the ORF of the gene (Fig. 3B; Supplemental Fig. S3A). Fine mapping of WUS-binding sites within the two regions was carried out by testing a series of short oligonucleotides for their ability to bind to WUS using electrophoretic mobility shift assays (EMSA) (Fig. 3D). This analysis revealed the presence of one WUS-binding element in the upstream region and two elements in the downstream region (Fig. 3D). A comparison of DNA sequences of both the upstream and downstream binding elements revealed the presence of a conserved TAAT core in all three (Fig. $3 \mathrm{E})$, which shares similarities with the WUS-binding element described for AGAMOUS (AG) (Lohmann et al. 2001). We confirmed the presence of WUS protein in the WUS-DNA complex by observing a "supershift" when we incubated $C L V 3$ promoter oligonucleotides representing the three binding sequences with anti-WUS antibody (Fig. 3D; Supplemental Fig. S4G-I). Introduction of single 
Yadav et al.

A

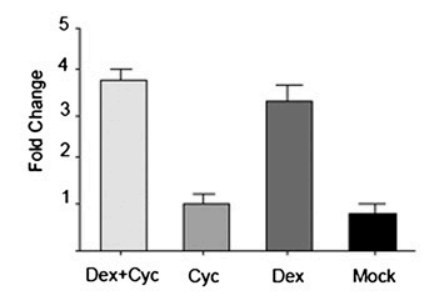

B

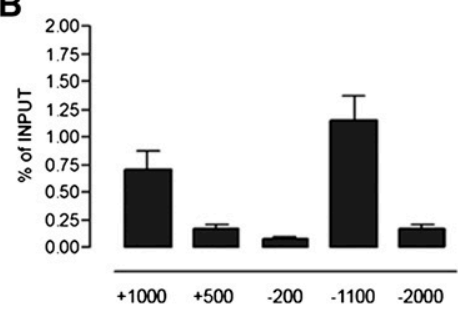

C

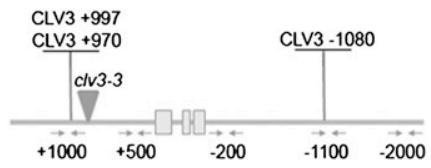

D

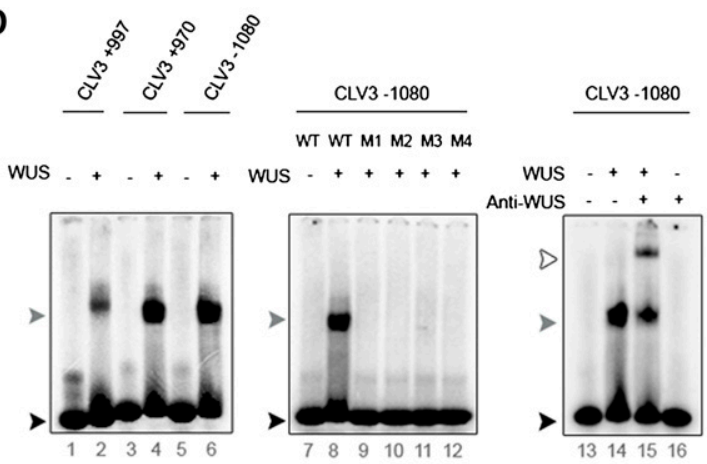

E

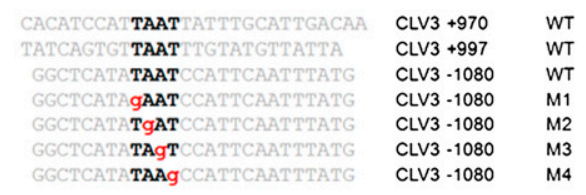

F

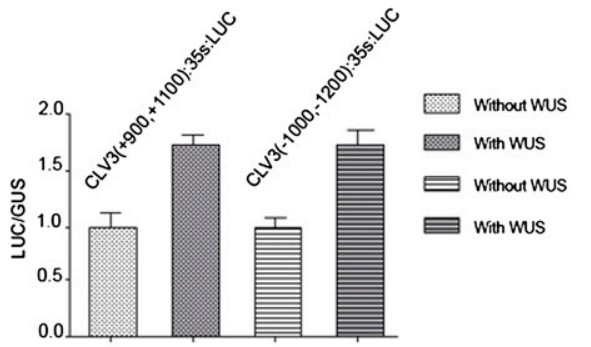

Figure 3. CLV3 is a direct transcriptional target of WUS. (A) qRT-PCR showing the increase in CLV3 transcripts upon activation of WUS by using a Dex-inducible system within $2 \mathrm{~h}$ of Dex application in the absence and presence of protein synthesis inhibitor Cyc. Error bars represent standard deviation. $(B)$ ChIP-qPCR showing relative enrichment of regulatory regions of CLV3. Regions are mapped with respect to transcription start site $(+1)$. (C) The clv3-3 T-DNA allele is located $55 \mathrm{nt}$ in front of the WUS-binding elements located downstream from the ORF. ( $D$, left panel, lanes 1-6) EMSA showing recombinant WUS protein bound to radiolabeled CLV3 oligonucleotides from the +970 and +997 downstream regions and from the -1080 upstream region. (Middle panel, lanes 7-12) Absence of binding of WUS to mutant forms of CLV3 (-1080)radiolabeled oligonucleotides in which each of the four bases (in red) within the TAAT core have been substituted. (Right panel, lanes 13-16) EMSA showing recombinant WUS protein bound to radiolabeled CLV3 oligonucleotides -1080 in the absence and presence of anti-WUS antibody A black arrowhead indicates free probe, a gray arrowhead indicates band shift, and a white arrowhead indicates "supershift." The sequences of wild-type and mutant form (M1-M4) oligonucleotides are indexed in $E$. $(F)$ Transient transfection assay plots showing activation of LUC reporter when cloned downstream from a 35S minimal promoter region containing the WUS-binding element found in the CLV3 promoter [CLV3(-1000,-1200):35s::LUC] and a region containing WUS-binding elements found in the CLV3 downstream region [CLV3(+900,+1100):35s:: LUC].

base pair mutation within the TAAT core of the WUSbinding site in the CLV3 promoter completely abolished WUS binding (Fig. 3D). Competition experiments using increasing concentrations of specific wild-type cold (Supplemental Fig. S4A-C) and mutated cold oligos (Supplemental Fig. S4D-F) demonstrated the specificity of binding of WUS to all three binding sites. We next tested whether the WUS-binding element in the CLV3 promoter is capable of activating transcription in a transient expression system by examining the LUCIFERASE (LUC) reporter expression levels in protoplasts isolated from mesophyll cells of leaves. These experiments revealed a WUS-dependent activation of $L U C$ when the CLV3 promoter contexts containing the WUS-binding elements were used to drive the LUC gene (Fig. 3F). The WUSdependent activation of $L U C$ was abolished when the CLV 3 promoter context carrying a mutated WUS-binding element was used (Supplemental Fig. S5). The loss-of-function CLV3 allele clv3-3 (a T-DNA insertion described in an earlier study) (Fletcher et al. 1999) is located $\sim 55$ nucleotides (nt) upstream of the 3' WUS-binding element, suggesting that it may have disrupted WUS-mediated transcriptional regulation. Moreover CLV3 promoter deletion covering the region containing downstream WUSbinding elements identified here has been shown to significantly reduce the promoter activity (Muller et al. 2006). Taken together, these results demonstrate that WUS binds to the genomic regions of $C L V 3$ to activate its transcription.

\section{Computational model explains the importance of maintaining WUS protein gradient}

Our results show that the expression of the WUS gene is restricted to a few cells of the RM/OC, while the WUS protein migrates into adjacent cells of the CZ to activate $C L V 3$ through a direct transcriptional control. To understand the importance of WUS protein migration into adjacent cells of the CZ to activate the CLV3 promoter in the context of the known stem cell regulatory network, we implemented a computational model of a three-dimensional (3D) SAM tissue (Supplemental Fig. S6; see the Supplemental Material). The model includes the direct activation 
of $C L V 3$ transcription from the WUS protein (Fig. 2B) and a negative regulation of WUS from a CLV3 signal that emanates from the CZ (Brand et al. 2000; Schoof et al. 2000). In addition, WUS is activated by a localized cytokinin signal (Jönsson et al. 2005; Gordon et al. 2010; Hohm et al. 2010), and a hypothetical signal originating from cells of the L1 layer is used as a cofactor for CLV3 activation (see the Supplemental Material; Jönsson et al. 2003). This minimal network was sufficient to reproduce the wildtype expression domains of CLV3 and WUS, as well as produce several perturbations (Supplemental Fig. S7). The perturbations include blocked migration of WUS protein (Fig. 4B), which results in meristem termination (Supplemental Fig. S1B,C), and also misexpression of WUS in cells of the $\mathrm{CZ}$ by using the $C L V 3$ promoter (Fig. $4 \mathrm{~B}$ ), which has been shown to result in expansion of the stem cell domain and CLV3 expression (Yadav et al. 2010; Brand et al. 2002).

Taken together, our work shows the importance of maintaining the WUS protein gradient in regulating stem cell number. In the absence of cell behavioral mechanisms such as asymmetric and oriented cell division and cell migration to fine-tune stem cell numbers, the self-repression of stem cell inducer through a direct transcriptional mechanism may allow quick resetting of the CLV-WUS feedback loop. Therefore, it is essential to understand the regulation of WUS protein movement together with the CLV3-mediated transcriptional repression of WUS. This will require an understanding of the structural properties of plasmodesmata, the symplasmic connectivity patterns of cells, signals intrinsic to the WUS protein, and extrin-

A
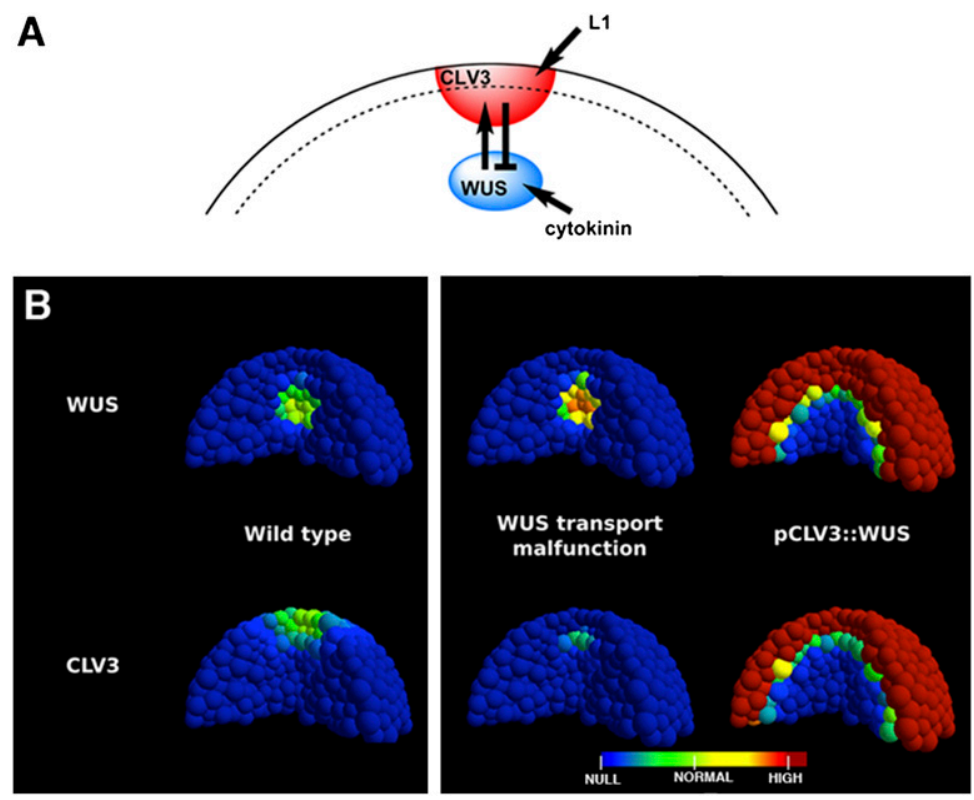

Figure 4. A computational model for $3 \mathrm{D}$ patterning of gene expressions in the SAM. (A) Illustration of the gene interactions in the model showing typical expression domains in the SAM. $(B)$ Simulation results from a CLV3-WUS feedback model (see the Supplemental Material). The top row shows WUS expression, and the bottom row shows CLV3 expression. The left panel shows the wild-type expression domains, the middle panel shows the results from a perturbed WUS diffusion (the disappearance of the CLV3 expression can be interpreted as a terminated meristem), and the right panel shows the expanded stem cell domain/CLV3 expression domain along the outer layers of cells upon misexpression of WUS from the CLV3 promoter, as shown in earlier studies (Brand et al. 2002; Yadav et al. 2010). In the color bar, a linear scale is used and "normal" represents the template value used for optimization (1; i.e., concentration value 1 in arbitrary units). sic signals such as plant hormone cytokinin, which has been implicated in stem cell homeostasis (Leibfried et al. 2005; Gordon et al. 2010). We observed that WUS protein migrates laterally into the differentiating progeny of stem cells. New studies are required to unravel WUS function in these cells, which may lead to better insights into the molecular mechanisms that regulate differentiation behavior and cell division patterns of stem cell progenitors, both of which are critical for stem cell maintenance.

\section{Materials and methods}

\section{Plant growth and live imaging}

Plant growth, live imaging, microscopic protocols, and phenotypic analysis have been performed as described earlier (Reddy et al. 2004; Reddy and Meyerowitz 2005; Yadav et al. 2009, 2010).

\section{Plasmid constructs, selection of transgenic lines, and rescue analysis}

The details of the 35S::WUS:GR construct have been published (Yadav et al. 2010). To generate the $p W U S: e G F P: W U S$ plasmid, eGFP was fused in-frame to the $\mathrm{N}$ terminus of the WUS ORF and introduced into the WUS promoter containing 5.6-kb upstream and 1.2-kb downstream regions between AscI and StuI in pCAMBIA2300 (Yadav et al. 2008). To generate nls-GFP-WUS, nlsGFP (kind gift from Dr. Ji Young-Lee) was fused in-frame with the $\mathrm{N}$ terminus of the WUS ORF and introduced into the WUS promoter. To generate 2xeGFP:WUS, two tandem repeats of $e G F P$ were fused in-frame with the $\mathrm{N}$ terminus of the WUS ORF and introduced into the WUS promoter. Transgenic lines were generated in the Landsberg erecta background.

To test the functionality of $p W U S:: e G F P: W U S$, it was introduced into the wus-1 $1^{-/+}$background. We generated $55 \mathrm{~T} 1$ lines. Through PCR-based genotyping and sequencing, we identified only $16 \mathrm{~T} 1$ lines of the wus- $1^{-/-}$or wus- $1^{-/+}$genotypes, which were phenotypically similar to wild-type plants. Since it was not possible to distinguish between wus-1 $1^{-/-}$or wus- $1^{-/+}$genotypes because of the eGFP:WUS transgene, we followed five independent $\mathrm{T} 1$ lines in subsequent generations. The progeny of five T2 lines that could be either wus $-1^{-/-}$or wus $-1^{-/+}$were subjected to phenotypic analysis in the T3 generation. All progeny in the T3 generation that carried the transgene showed complete rescue and resembled wild-type plants (Supplemental Fig. S1A). Two independent lines were followed until the T4 generation to establish a homozygous wus- $1^{-/}$line that is phenotypically similar to wild type. To test the functionality of $p W U S:: n l s-G F P: W U S$, we generated $47 \mathrm{~T} 1$ lines. We identified only seven lines that could be either wus-1 $1^{-/-}$or wus- $1^{-/+}$, out of which two lines showed wus-1-like phenotype. To evaluate the functionality of the nlsGFP:WUS protein further, we followed five T1 lines of the wus- $1^{+/-}$ genotype in subsequent generations. The T2 progeny of three independent lines that carried the transgene segregated wus-like phenotype (Supplemental Fig. S1B). The T2 progeny of the remaining two lines occasionally produced secondary shoots that terminated after making four to six wild-type-like flowers that set seeds, suggesting that nls-GFP:WUS can partially rescue secondary shoots, whereas it can completely rescue FM phenotypes (Supplemental Fig. S1D). In the case of $p W U S:: 2 X G F P: W U S$, we generated 27 independent $\mathrm{T} 1$ lines. We identified only seven $\mathrm{T} 1$ lines that could be either wus- $1^{-/-}$or wus- $1^{-/+}$, and five of these lines exhibited wus-like phenotype (Supplemental Fig. S1C). The $\mathrm{T} 2$ progeny of the remaining two lines that carried the transgene segregated wus-1-like phenotype or produced occasional secondary shoots with few wild-type flowers before terminating (Supplemental Fig. S1E).

\section{Acknowledgments}

We thank Frans Tax and Patricia Springer for comments on the manuscript. Live-imaging work was done at the microscopy core 
facility of the Center for Plant Cell Biology (CEPCEB), and genomics work was done at the Institute of Integrative Genome Biology (IIGB), UCR. This work was funded by National Science Foundation grant IOS-0718046 to G.V.R.

\section{References}

Brand U, Fletcher JC, Hobe M, Meyerowitz EM, Simon R. 2000. Dependence of stem cell fate in Arabidopsis on a feedback loop regulated by CLV3 activity. Science 289: 617-619.

Brand U, Grunewald M, Hobe M, Simon R. 2002. Regulation of CLV3 expression by two homeobox genes in Arabidopsis. Plant Physiol 29: 565-575.

Clark SE, Williams RW, Meyerowitz EM. 1997. The CLAVATA1 gene encodes a putative receptor kinase that controls shoot and floral meristem size in Arabidopsis. Cell 89: 575-585.

Crawford KM, Zambryski PC. 2000. Subcellular localization determines the availability of non-targeted proteins to plasmodesmatal transport. Curr Biol 10: 1032-1040.

Fletcher JC, Brand U, Running MP, Simon R, Meyerowitz EM. 1999. Signaling of cell fate decisions by CLAVATA3 in Arabidopsis shoot meristems. Science 283: 1911-1914.

Gallagher KL, Benfey PN. 2005. Not just another hole in the wall: understanding intercellular protein trafficking. Genes Dev 19: 189195.

Gallagher KL, Paquette AJ, Nakajima K, Benfey PN. 2004. Mechanisms regulating SHORT-ROOT intercellular movement. Curr Biol 14: $1847-1851$.

Gordon SP, Chickarmane VS, Ohno C, Meyerowitz EM. 2010. Multiple feedback loops through cytokinin signaling control stem cell number within the Arabidopsis shoot meristem. Proc Natl Acad Sci 106: 16529-16534.

Hohm T, Zitzler E, Simon R. 2010. A dynamic model for stem cell homeostasis and patterning in Arabidopsis meristems. PLoS ONE 5: e9189. doi: 10.1371/jounrla.pone.0009189.

Jönsson H, Shapiro BE, Meyerowitz EM, Mjolsness E. 2003. Signalling in multicellular models of plant development. In On growth, form, and computers (ed. S Kumar, P Bentley), pp. 156-161. Academic Press, London.

Jönsson H, Heisler M, Reddy GV, Agrawal V, Gor V, Shapiro BE, Mjolsness E, Meyerowitz EM. 2005. Modeling the organization of the WUSCHEL expression domain in the shoot apical meristem. Bioinformatics 21: i232-i240. doi: 10.1093/bioinformatics/bti1036.

Kinoshita A, Betsuyaku S, Osakabe Y, Mizuno S, Nagawa S, Stahl Y, Simon R, Yamaguchi-Shinozaki K, Fukuda H, Sawa S. 2010. RPK2 is an essential receptor-like kinase that transmits the CLV3 signal in Arabidopsis. Development 137: 3911-3920.

Kondo T, Sawa S, Kinoshita A, Mizuno S, Kakimoto T, Fukuda H, Sakagami Y. 2006. A plant peptide encoded by CLV3 identified by in situ MALDI-TOF MS analysis. Science 313: 845-848.

Laux T, Mayer KF, Berger J, Jurgens G. 1996. The WUSCHEL gene is required for shoot and floral meristem integrity in Arabidopsis. Development 122: 87-96.

Leibfried A, To JP, Busch W, Stehling S, Kehle A, Demar M, Kieber JJ, Lohmann JU. 2005. WUSCHEL controls meristem function by direct regulation of cytokinin-inducible response regulators. Nature 438: 1172-1175.

Lohmann JU, Hong RL, Hobe M, Busch MA, Parcy F, Simon R, Weigel D. 2001. A molecular link between stem cell regulation and floral patterning in Arabidopsis. Cell 105: 793-803.

Mayer KF, Schoof H, Haecker A, Lenhard M, Jürgens G, Laux T. 1998. Role of WUSCHEL in regulating stem cell fate in the Arabidopsis shoot meristem. Cell 95: 805-815.

Muller R, Borghi L, Kwiatkowska D, Laufs P, Simon R. 2006. Dynamic and compensatory responses of Arabidopsis shoot and floral meristems to CLV3 signaling. Plant Cell 18: 1188-1198.

Müller R, Bleckmann A, Simon R. 2008. The receptor kinase CORYNE of Arabidopsis transmits the stem cell-limiting signal CLAVATA3 independently of CLAVATA1. Plant Cell 20: 934-946.

Ogawa M, Shinohara H, Sakagami Y, Matsubayashi Y. 2008. Arabidopsis CLV3 peptide directly binds CLV1 ectodomain. Science 319: 294.

Reddy GV. 2008. Live-imaging stem cell homeostasis in the Arabidopsis shoot apex. Curr Opin Plant Biol 11: 88-93.
Reddy GV, Meyerowitz EM. 2005. Stem cell homeostasis and growth dynamics can be uncoupled in the Arabidopsis shoot apex. Science 310: 663-667.

Reddy GV, Heisler MG, Ehrhardt DW, Meyerowitz EM. 2004. Real-time lineage analysis reveals oriented cell divisions associated with morphogenesis at the shoot apex of Arabidopsis thaliana. Development 131: 4225-4237.

Rieu I, Laux T. 2009. Signaling pathways maintaining stem cells at the plant shoot apex. Semin Cell Dev Biol 20: 1083-1088.

Schoof H, Lenhard M, Haecker A, Mayer KF, Jürgens G, Laux T. 2000. The stem cell population of Arabidopsis shoot meristems in maintained by a regulatory loop between the CLAVATA and WUSCHEL genes. Cell 100: 635-644.

Spradling AC, Nystul T, Lighthouse D, Morris L, Fox D, Cox R, Tootle T, Frederick R, Skora A. 2008. Stem cells and their niches: integrated units that maintain Drosophila tissues. Cold Spring Harb Symp Quant Biol 73: 49-57.

Yadav RK, Fulton L, Batoux M, Schneitz K. 2008. The Arabidopsis receptor-like kinase STRUBBELIG mediates inter-cell-layer signaling during floral development. Dev Biol 323: 261-270.

Yadav RK, Girke T, Pasala S, Xie M, Reddy GV. 2009. Gene expression map of the Arabidopsis shoot apical meristem stem cell niche. Proc Nat1 Acad Sci 106: 4941-4946.

Yadav RK, Tavakkoli M, Reddy GV. 2010. WUSCHEL mediates stem-cell homeostasis by regulating stem-cell number and patterns of cell division and differentiation of stem-cell progenitors. Development 137: 3581-3589. 


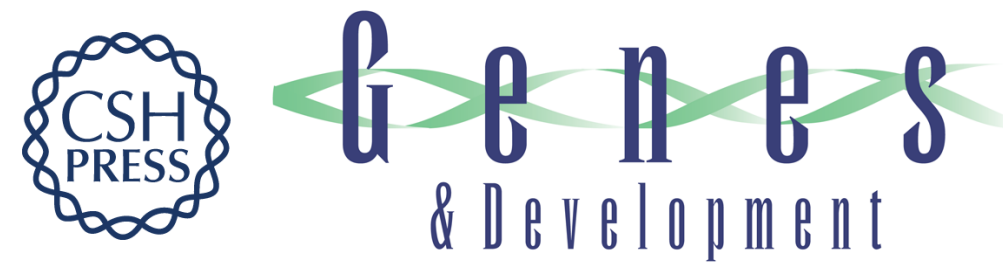

\section{WUSCHEL protein movement mediates stem cell homeostasis in the Arabidopsis shoot apex}

Ram Kishor Yadav, Mariano Perales, Jérémy Gruel, et al.

Genes Dev. 2011, 25:

Access the most recent version at doi:10.1101/gad.17258511

\section{Supplemental http://genesdev.cshlp.org/content/suppl/2011/10/06/25.19.2025.DC1 Material}

References This article cites 28 articles, 14 of which can be accessed free at: http://genesdev.cshlp.org/content/25/19/2025.full.html\#ref-list-1

\section{License}

Email Alerting

Receive free email alerts when new articles cite this article - sign up in the box at the top Service 